\title{
Efectividad del uso del mate de coca en la reducción del estrés laboral en administrativos de una universidad
}

\author{
María Teresa Rivera-Encinas ${ }^{1,2}$
}

RESUMEN

Objetivo: Evaluar la efectividad del uso del mate de coca en la reducción del estrés laboral en administrativos de una universidad y determinar sus principales características sociodemográficas.

Material y métodos: Se aplicó el cuestionario para la detección del estrés laboral de la OIT/OMS a dos grupos de trabajadores, experimental (10) y control (10), empleando randomización simple. Se hizo un experimento cruzado en 31 días, utilizando mate de coca y agua para cada grupo. Se utilizó el análisis de Wilcoxon para comparar los grupos antes y después, y la prueba U de Mann Whitnney para comparar los grupos experimental y control.

Resultados: Se encontró disminución en el puntaje de estrés laboral en el grupo que usó mate de coca (de 71,2 a 68,6), pero no fue estadísticamente significativo $(p>0,05)$. Las características de los administrativos con estrés laboral fueron: $55 \%$, de sexo femenino; el $60 \%$ tuvo un tiempo de servicio mayor o igual a 5 años; el $90 \%$ trabajó más de 8 horas al día; el $25 \%$ realizaba meditación y $60 \%$ realizaba actividad física.

Conclusiones: El mate de coca no es efectivo en la reducción del estrés laboral. Se necesitan más estudios al respecto. Se recomienda fomentar la salud mental en el trabajo, utilizando intervenciones y desarrollando guías para la reducción del estrés laboral.

Palabras clave: estrés laboral, mate de coca, administrativos.

\section{Effectiveness of coca tea to reduce work stress in the administrative staff in a university}

\section{ABSTRACT}

Objetive: To evaluate the effectiveness of coca tea to reduce work stress in the administrative staff in a university.

Material and methods: The questionnaire for the detection of work stress of the ILO / WHO was applied to two groups of workers, experimental (10) and control (10) using a simple randomization. A cross over experiment was done in 31 days using coca tea and water for each group. Wilcoxon analysis was used to compare groups before and after the test, and the $U$ Mann Whitnney test was used to compare the experimental and control groups.

Results: Decrease of work stress was found in the score of the group who drank coca tea (from 71,2 to 68,6 ), but was not statistically significant $(p>0,05)$. Administrative staff features with job stress were: $55 \%$ female; $60 \%$ had 5 years or more of service; $90 \%$ worked more than 8 hours per day; $25 \%$ performed meditation and $60 \%$ performed physical activity.

Conclusions: Tea coca is not effective to reduce work stress. More studies are needed. To improve mental health at work is recommended to use interventions and making guides to reduce work stress.

Key words: work stress, coca tea, and administrative staff.

1. Máster en Salud Pública con mención en Salud Ocupacional. Facultad de Medicina Humana de la Universidad de San Martín de Porres. Lima - Perú.

2. Médica residente de psiquiatría del Instituto Nacional de Salud Mental “Honorio Delgado - Hideyo Noguchi”. 


\section{INTRODUCCIÓN}

En diferentes ámbitos, los seres humanos realizan actividades que demandan energía y generan estrés, desde la realización de un ejercicio físico hasta la utilización de funciones mentales superiores como la atención, memoria, abstracción, entre otras. Según Sandín, el estrés es la respuesta en términos de activación del eje hipotálamo-hipófisocórticosuprarrenal, con elevación de la secreción de corticoides (cortisol, etc.), y del eje simpáticomédulo-suprarrenal, como reacción a estímulos inespecíficos (estresores) ${ }^{(1)}$. Para Ivancevich, el estrés es una respuesta adaptativa, mediada por las características individuales $\mathrm{y} / \mathrm{o}$ por procesos psicológicos, la cual es a la vez consecuencia de alguna acción, de una situación o un evento externo que plantean a la persona especiales demandas física y/o psicológicas ${ }^{(2)}$.

Se conoce que cuando el cuerpo es sometido a un estímulo ocurren respuestas constituidas en tres etapas:

1. La reacción de alarma, demuestra que toda exposición a estímulos (estresores) externos (ambiente laboral) o internos (fisiológicos/ tensión o psicológicos/ responsabilidades), producen cambios adecuados y necesarios de adaptación inmediata y transitoria. Esta reacción acaba rápidamente cuando el estímulo o estresor desaparece.

2. Si el estímulo persiste, el organismo puede desarrollar resistencia, la cual ayuda durante un tiempo a tolerar las tensiones. Sin embargo, dicha resistencia no le sirve indefinidamente porque puede disminuir o agotar sus reservas bioquímicas.

\section{A medida que dichas sustancias van disminuyendo comienza la fase de agotamiento ${ }^{(3)}$.}

De igual modo, se pueden transpolar estos conceptos al ámbito ocupacional, por considerar al estrés como un factor de riesgo psicosocial. Según la OMS, el estrés laboral "es la reacción que puede tener el individuo ante exigencias y presiones laborales que no se ajustan a sus conocimientos y capacidades, y que ponen a prueba su capacidad para afrontar dichas situaciones", siendo el resultado del desequilibrio entre las demandas del ambiente (estresores externos e internos) y los recursos disponibles del sujeto en el lugar de trabajo, los que influyen en el trabajador y pueden afectar la salud del mismo ${ }^{(4)}$.
Desde el punto de vista legal, en conformidad con el artículo 103 del Reglamento de la Ley de Salud y Seguridad del Trabajo del Perú, se considera que existe exposición a los riesgos psicosociales cuando se perjudica la salud de los trabajadores, causando estrés y, a largo plazo, una serie de sintomatologías clínicas como enfermedades cardiovasculares, respiratorias, inmunitarias, gastrointestinales, dermatológicas, endocrinológicas, músculo esqueléticas, mentales, entre otras ${ }^{(5)}$.

Existen datos alarmantes, como el de la Encuesta Nacional de Seguridad e Higiene en el Trabajo del 2007, realizada por el Instituto Nacional de Salud e Higiene en el Trabajo de España, el cual refiere que el $22,5 \%$ de los trabajadores consideran que el trabajo afecta negativamente su salud, atribuyendo que sus dolencias más frecuentes son el dolor de espalda en el $13 \%$ de encuestados, dolor de cuello $6,3 \%$ y el estrés en el 6,3\% (INSHT 2009) ${ }^{(6)}$. Esto ilustra la magnitud del problema, el cual se cree puede ser proporcionalmente más significativo cuando se trata de personal en constante tensión, como es el caso de los administrativos de una universidad, por los documentos que deben realizar, tiempos límites de entrega, carga laboral, entre otros; con los que deben de lidiar usualmente, motivo por el que son considerados como población vulnerable y en riesgo de sufrir de estrés laboral dentro de estos escenarios. Asimismo, se relaciona con la baja productividad, alto ausentismo de los trabajadores, malos tratos contra los mismos compañeros y público en general, por lo que las empresas se ven perjudicadas por el menor ingreso económico y desprestigio ante estos hechos, totalmente prevenibles ${ }^{(7)}$. En el informe del National Institute for Occupational Safety and Health (NIOSH), entre el 28 al $40 \%$ de los trabajadores señalaron que su trabajo resultaba estresante; y que aquellos trabajadores con ansiedad, estrés, o alteraciones neuróticas perdieron muchos más días de trabajo (25 días de promedio perdidos por trabajador) en el año 2001, que aquellos que no presentaban este tipo de alteraciones (solo seis días como promedio perdidos por trabajador $)^{(8)}$.

En el Perú, son varios los profesionales que realizan funciones ejecutivas y administrativas que sufren de este mal. En un estudio con 138 fiscales y 149 jueces, en Lima - Perú, en el que luego de aplicarles un cuestionario compuesto por cuatro instrumentos para determinar los niveles de estrés general y laboral, ansiedad y depresión, se encontró que un cuarto de los encuestados, presentaban altos niveles de estrés, ansiedad y depresión ${ }^{(9)}$. 
Se conocen diferentes formas de contrarrestar el estrés laboral, que van desde lo lógico y racional, hasta lo innovador y surrealista. Al fortalecer la equidad de las relaciones del jefe y sus trabajadores, reconocer el trabajo realizado(10), establecer programas de intervención y prevención del estrés, ansiedad y depresión ${ }^{(9,11)}$ y emplear la psicología ocupacional ${ }^{(8)}$, se podrían prevenir enfermedades y accidentes laborales relacionados con los riesgos psicosociales, por parte de algunos gobiernos ${ }^{(8)}$. En Japón, el Ministerio de Salud recomienda que todos los trabajadores, sin diferencia de edad, duerman una siesta de por lo menos 30 minutos durante su trabajo en la tarde, por lo que varias empresas han comenzado a implementar habitaciones para dicho uso. En la investigación de Takahashi, se describe que dormir incluso durante el trabajo mejora el rendimiento de los trabajadores ${ }^{(12)}$.

Las políticas de salud ocupacional en Perú son aún austeras, por lo que implementar tales ejemplos, por ahora, serían poco factibles por casi todos los empleadores. Sin embargo, desde el punto de vista de la medicina tradicional, existen alternativas, como el uso de la hoja de coca, como posible intervención en la reducción del estrés laboral, puesto que empíricamente, se la utiliza para distintas dolencias, entre la que destacan la fatiga laboral y el estrés ${ }^{(13)}$.

Con la frase del célebre médico y gran político peruano don Hipólito Unanue en 1794: "La coca fue, entre nuestros sabios, lo que la manzana de la discordia entre los dioses", se resumen las diferentes discusiones que esta planta sagrada de los Incas había ocasionado hasta entonces en todos los círculos científicos, oficiales y políticos del Perú, y que hasta ahora persisten ${ }^{(14)}$.

Según Fernando Cabieses, la infusión de coca como una forma culturalmente aceptada, es parte del uso tradicional, por lo que se justifica industrializar la hoja de coca en forma de bolsitas filtrantes y, ser de esta manera, el uso legal más extendido en nuestro país ${ }^{(15)}$.

El principal componente de la hoja de coca es la cocaína, una base débil con un pKa de 8,6. Los efectos dependen de la farmacocinética y farmacodinamia de la sustancia, así como de la vía de administración ${ }^{(16)}$. En la tabla 1 se comparan las diferentes formas de abuso de la cocaína y las principales características farmacológicas según su uso.
La literatura señala que los usuarios de cocaína oral no presentan trastornos significativos de las funciones vitales (presión, pulso, respiración), clínicas, hematológicas, ni conducta adictiva patológica y más bien, dan respuestas favorables para compensar el estrés ${ }^{(17,18,19)}$.

Por vía oral, como la consumen los chacchadores y los bebedores de infusiones/mates, la cocaína se absorbe muy lentamente por el intestino, dependiendo de varios factores, como de la preparación usada, del contenido de las hojas y de la presencia o ausencia de sustancias alcalinas en la boca del masticador. Sin embargo, cuando llega al hígado se destruye muy rápidamente, por lo que nunca alcanza velocidades de ingreso ni concentraciones plasmáticas lo suficientemente altas como para excitar al consumidor ${ }^{(8,14,16)}$.

Así pues, estudios de farmacocinética de la cocaína revelan:

a) La cocaína, al ingresar al organismo por vía nasal, pulmonar o inyección venosa, muscular o subcutánea, pasa directamente a la sangre y actúa de inmediato sobre el sistema nervioso al atravesar la barrera hematoencefálica ${ }^{(14)}$.

b) La cocaína que ingresa por vía digestiva no pasa a la sangre directamente. Realiza el conocido Fenómeno de Primer paso, que es cuando lo ingerido es metabolizado por el hígado y se inactiva gran parte de la molécula, de tal manera que solo un pequeño porcentaje entra a la sangre $^{(14)}$.

c) La cocaína que está en la sangre es destruida por la enzima colinesterasa $\mathrm{B}^{(14,16)}$.

d) Los productos de desecho son eliminados por el riñón, por lo que pueden ser detectados en orina ${ }^{(14)}$. 
Tabla 1. Formas de abuso de la cocaína

\begin{tabular}{|c|c|c|c|c|c|c|c|}
\hline $\begin{array}{l}\text { Tipo de } \\
\text { sustancia }\end{array}$ & $\begin{array}{l}\text { Concentración } \\
\text { de cocaína }\end{array}$ & $\begin{array}{c}\text { Vía de } \\
\text { administración }\end{array}$ & $\begin{array}{c}\% \text { en } \\
\text { plasma }\end{array}$ & $\begin{array}{c}\text { Velocidad de } \\
\text { aparición de } \\
\text { efectos }\end{array}$ & $\begin{array}{c}\text { Concentración } \\
\text { máxima } \\
\text { plasmática }\end{array}$ & $\begin{array}{c}\text { Duración } \\
\text { de } \\
\text { efectos }\end{array}$ & $\begin{array}{c}\text { Desarrollo } \\
\text { de } \\
\text { dependencia }\end{array}$ \\
\hline $\begin{array}{l}\text { Hojas de } \\
\text { coca }\end{array}$ & $0,5-1,5 \%$ & $\begin{array}{l}\text { Mascado } \\
\text { infusión oral }\end{array}$ & $\begin{array}{l}20- \\
30 \%\end{array}$ & LENTA & $\begin{array}{c}60 \\
\text { minutos }\end{array}$ & $\begin{array}{c}30-60 \\
\text { minutos }\end{array}$ & NO \\
\hline $\begin{array}{l}\text { Clorhid. } \\
\text { Cocaína }\end{array}$ & $12-75 \%$ & $\begin{array}{l}\text { Tópica: ocular, } \\
\text { genital, } \\
\text { intranasal } \\
\text { (esnifar) }\end{array}$ & $\begin{array}{l}20- \\
30 \%\end{array}$ & $\begin{array}{l}\text { RELATIV. } \\
\text { RÁPIDA }\end{array}$ & $\begin{array}{c}5-10 \\
\text { minutos }\end{array}$ & $\begin{array}{l}30-60 \\
\text { minutos }\end{array}$ & $\begin{array}{c}\text { sí } \\
\text { LARGO } \\
\text { PLAZO }\end{array}$ \\
\hline $\begin{array}{l}\text { Clorhid. } \\
\text { Cocaína }\end{array}$ & $12-75 \%$ & $\begin{array}{l}\text { Parenteral: } \\
\text { endovenosa, } \\
\text { subcutánea, } \\
\text { intramuscular }\end{array}$ & $100 \%$ & RÁPIDA & $\begin{array}{c}30-45 \\
\text { segundos }\end{array}$ & $\begin{array}{l}10-20 \\
\text { minutos }\end{array}$ & $\begin{array}{c}\text { sí } \\
\text { CORTO } \\
\text { PLAZO }\end{array}$ \\
\hline $\begin{array}{l}\text { Pasta de } \\
\text { coca }\end{array}$ & $\begin{array}{c}40-85 \% \\
\text { (Sulfato de } \\
\text { cocaína) }\end{array}$ & Fumada & $\begin{array}{l}70- \\
80 \%\end{array}$ & $\begin{array}{c}\text { MUY } \\
\text { RÁPIDA }\end{array}$ & $\begin{array}{c}8-10 \\
\text { segundos }\end{array}$ & $\begin{array}{l}5-10 \\
\text { minutos }\end{array}$ & $\begin{array}{l}\text { Sí CORTO } \\
\text { PLAZO }\end{array}$ \\
\hline $\begin{array}{l}\text { Cocaína } \\
\text { base }\end{array}$ & $\begin{array}{l}30-80 \% \\
\text { (alcaloide } \\
\text { cocaína) }\end{array}$ & $\begin{array}{l}\text { Inhalada } \\
\text { fumada }\end{array}$ & $\begin{array}{l}70- \\
80 \%\end{array}$ & $\begin{array}{c}\text { MUY } \\
\text { RÁPIDA }\end{array}$ & $\begin{array}{c}8-10 \\
\text { segundos }\end{array}$ & $\begin{array}{c}5-10 \\
\text { minutos }\end{array}$ & $\begin{array}{l}\text { sí CORTO } \\
\text { PLAZO }\end{array}$ \\
\hline
\end{tabular}

Referencia: Lizasoain y et al. Cocaína: aspectos farmacológicos. Rev. Adicciones 2002.

Los niveles de benzoilecgonina (metabolito de la cocaína) en orina, entre 2000 y $5000 \mathrm{ng} / \mathrm{ml}$ se pueden encontrar luego de ingerir una taza de mate preparada con 1 gramo de hojas molidas ${ }^{(20)}$. Esto es considerado mucho menor a lo encontrado en chacchadores crónicos tradicionales los que pueden llegar a $200000 \mathrm{ng} / \mathrm{ml}$; incluso tan mínimo en comparación de los consumidores habituales de clorhidrato de cocaína por vía nasal que pueden situarse entre 20000 y $200000 \mathrm{ng} / \mathrm{ml} \mathrm{de}$ benzoilecgonina ${ }^{(13)}$.

En estudios utilizando mate de coca, se han usado bolsitas filtrantes de hojas, porque son las únicas unidades industrialmente pesadas y homogeneizadas (1 gramo de un gramo de hoja de coca naturales molidas). El mate o infusión se prepara colocando una bolsita filtrante en una taza de $200 \mathrm{ml}$ de agua caliente, aproximadamente durante tres minutos, liberándose así cocaína contenida en las hojas secas ${ }^{(21)}$. De esta forma, no tiene acción excitante, embriagadora ni genera dependencia ${ }^{(17-20)}$.

El reconocimiento de la presencia de estrés laboral dentro una universidad, la Universidad de San Martín de Porres (USMP), por ser un lugar lleno de actividades para los diferentes trabajadores administrativos, que son vulnerables a este hecho por la carga de trabajo, fue punto de atención, ya que no se han realizado estudios previos, de tal modo, que resultó el presente trabajo ser original. La importancia radicó en conocer la magnitud de estrés laboral en los administrativos y tomar medidas seguras que lo reduzcan, usando mate de coca, el cual resulta dentro de la medicina tradicional y apoyo intercultural, un medio bueno y económico, que la USMP podría adoptar entre sus trabajadores, con el fin de mejorar el clima organizacional, la atención al usuario y el prestigio dentro y fuera de la institución.

El objetivo del presente estudio fue evaluar la efectividad del uso del mate de coca en la reducción el estrés laboral en administrativos de la Facultad de Medicina Humana (FMH) de la Universidad de San Martín de Porres- Lima, en el Semestre 2014 - II.

\section{MATERIAL Y MÉTODOS}

Se diseñó un estudio de corte analítico, prospectivo, longitudinal y experimental, que se realizó en la Facultad de Medicina Humana de la Universidad de San Martín de Porres, Lima, en el Semestre 2014 - II, teniendo la autorización del decano y del Comité de Ética de la universidad, previa solicitud.

La unidad de análisis fue el administrativo de la FMH USMP Lima, en el Semestre 2014 - II con estrés laboral, con puntaje mayor o igual a 51 en el 
cuestionario de la OIT/OMS para la detección de estrés laboral y que firmaron el consentimiento informado. Los criterios de exclusión considerados fueron los administrativos que tomaban fármacos ansiolíticos y/o antidepresivos, que programaron sus vacaciones durante el tiempo de seguimiento.

Se aplicó a todos los administrativos de la institución ( $n=71)$ contratados en el semestre 2014 - II, el cuestionario para determinar estrés laboral de la OIT / OMS. Este instrumento fue sustentado por Ivancevich y Matteson y tiene una confiabilidad de 0,966 , según el alfa de Cronbach, considerado como un nivel alto. Ha sido validado y utilizado en anteriores estudios en el mundo y en el Perú ${ }^{(22)}$. Su utilidad se encuentra en la detección del estrés laboral y la capacidad de predecir las fuentes de riesgos psicosociales ${ }^{(23)}$.

Este instrumento cuenta con 25 preguntas relacionadas a la estructura y clima organizacional, tecnología, influencia del líder, territorio y cohesión del grupo de trabajo, que se respondieron con un puntaje del 1 al 7. Se sumó aritméticamente el puntaje obtenido, se categorizaron a los sujetos con y sin estrés laboral en base al valor de la mediana, que fue 51 , en vista que la distribución de los datos fue heterogénea y dispersa.

De los 71 administrativos contratados en el período, 34 obtuvieron los puntajes que determinaban la presencia de estrés laboral. De ellos, 20 cumplieron con los criterios de inclusión y exclusión. A estos sujetos se les hizo una charla de información sobre el objetivo de estudio, la metodología y se les sensibilizó para afianzar el compromiso y la adherencia a la investigación, para que incluso, los días que no tuvieran supervisión directa, siguieran la indicación dada.

Las variables de interés fueron: estrés laboral, meditación, actividad física, tiempo promedio de trabajo, tiempo de servicio, edad y sexo.

Se los dividió en dos grupos, experimento o de interés y control o placebo, con el mismo número de sujetos, utilizando randomización simple.

El tiempo de estudio fue de 31 días, dividido en dos fases. El primer grupo experimento $(n=10)$, recibió mate de coca, preparado de la siguiente manera: en una taza de $200 \mathrm{ml}$ de agua caliente, se colocó una bolsita filtrante de mate de coca Herbi, durante tres minutos; mientras que el grupo control $(n=10)$, tomó un vaso de agua de $200 \mathrm{ml}$.

Este procedimiento se realizó durante 15 fechas continuas, incluidos fines de semana. Luego, en el día 16, considerado como período de lavado (wash out), no recibieron nada y se les aplicó el cuestionario. A partir del día 17, se realizó el cruce de los grupos (cross over), por lo que los que tomaron mate de coca, comenzaron a beber un vaso de agua de $200 \mathrm{ml}$. Así mismo, el otro grupo, recibió mate de coca, preparado de la manera descrita anteriormente. Ambos grupos ingirieron las bebidas por 15 fechas más. Finalmente, se les aplicó nuevamente el instrumento.

Se les hizo seguimiento con la ayuda de una lista chequeo y colaboradores capacitados. El investigador principal no intervino durante el experimento, siendo por ello simple ciego. Dos individuos abandonaron el estudio: uno de ellos, presentó al segundo día cefalea tipo migraña, por lo que decidió retirarse; mientras que el otro, cesó de forma intempestiva su trabajo en la institución en la mitad del estudio, por lo que descontinuó la investigación.

Para el análisis estadístico se utilizó el software SPSS v 21.0 (USD, Inc., Stone Mountain, Georgia, EE.UU). Las variables cuantitativas fueron resumidas de acuerdo a su media, desviación estándar, valores máximo y mínimo; y las variables categóricas se les asignó un valor, volviéndolas cuantitativas para analizarlas.

Se utilizó el test de Wilcoxon, para evaluar la variación antes y después dentro de cada grupo experimental; así como la prueba $U$ de Mann Withney para comparar los grupos experimento y control.

\section{RESULTADOS}

Se observó que el porcentaje de estrés laboral en la población de administrativos de la Facultad de Medicina Humana de la USMP fue de 47,9\% (Tabla 2).

Tabla 2. Estrés laboral en administrativos de la FMH de la USMP Lima, en el semestre 2014 - II

\begin{tabular}{ccc}
\hline $\begin{array}{c}\text { Estrés } \\
\text { laboral }\end{array}$ & $\mathrm{N}^{\circ}$ & $\%$ \\
\hline Sí & 34 & 47,9 \\
No & 37 & 52,1 \\
\hline Total & 71 & 100,0 \\
\hline
\end{tabular}


Luego de aplicarse los criterios de inclusión y exclusión, se consideraron 20 individuos. Se muestra en la tabla 3 las características sociodemográficas de la población de estudio.

Tabla 3. Características sociodemográficas

\begin{tabular}{|c|c|c|}
\hline \multicolumn{3}{|c|}{$\begin{array}{l}\text { Características } \\
\text { sociodemográficas }\end{array}$} \\
\hline Sexo & $\mathrm{N}^{\circ}$ & $\%$ \\
\hline $\begin{array}{l}\text { Masculino } \\
\text { Femenino }\end{array}$ & $\begin{array}{r}9 \\
11\end{array}$ & $\begin{array}{l}45,0 \\
55,0\end{array}$ \\
\hline $\begin{array}{c}\text { Total } \\
\text { Edades }\end{array}$ & $\begin{array}{l}20 \\
N^{\circ}\end{array}$ & $\begin{array}{c}100,0 \\
\%\end{array}$ \\
\hline $\begin{array}{c}20 \text { a } 40 \text { años } \\
41 \text { a } 50 \text { años } \\
51 \text { a más }\end{array}$ & $\begin{array}{l}9 \\
5 \\
6\end{array}$ & $\begin{array}{l}45,0 \\
25,0 \\
30,0\end{array}$ \\
\hline $\begin{array}{c}\text { Total } \\
\text { Tiempo de }\end{array}$ & $\begin{array}{l}20 \\
N^{\circ}\end{array}$ & $\begin{array}{c}100,0 \\
\%\end{array}$ \\
\hline $\begin{array}{l}\text { servicio } \\
<5 \text { años } \\
>=5 \text { años }\end{array}$ & $\begin{array}{r}8 \\
12\end{array}$ & $\begin{array}{l}40,0 \\
60,0\end{array}$ \\
\hline Total & 20 & 100,0 \\
\hline $\begin{array}{c}\text { Tiempo promedio } \\
\text { de trabajo }\end{array}$ & $\mathrm{N}^{\circ}$ & $\%$ \\
\hline $\begin{array}{l}1-8 \text { horas } \\
>8 \text { horas }\end{array}$ & $\begin{array}{r}2 \\
18\end{array}$ & $\begin{array}{l}10,0 \\
90,0\end{array}$ \\
\hline Total & 20 & 100,0 \\
\hline Actividad física & $\mathrm{N}^{\circ}$ & $\%$ \\
\hline $\begin{array}{l}\text { Sí } \\
\text { No }\end{array}$ & $\begin{array}{r}12 \\
8\end{array}$ & $\begin{array}{l}60,0 \\
40,0\end{array}$ \\
\hline Total & 20 & 100,0 \\
\hline Meditación & $\mathrm{N}^{\circ}$ & $\%$ \\
\hline $\begin{array}{l}\text { Sí } \\
\text { No }\end{array}$ & $\begin{array}{r}5 \\
15\end{array}$ & $\begin{array}{l}25,0 \\
75,0\end{array}$ \\
\hline Total & 20 & 100,0 \\
\hline
\end{tabular}

En la tabla 4, 5 y 6 se muestran los resultados, luego del experimento.

Tabla 4. Nivel de estrés luego del uso del mate de coca

\begin{tabular}{ccc}
\hline $\begin{array}{c}\text { Nivel de } \\
\text { estrés luego } \\
\text { del uso del } \\
\text { mate de coca }\end{array}$ & $\mathbf{N}^{\circ}$ & $\%$ \\
\hline Aumentó & 9 & 50,0 \\
Igual & 1 & 5,6 \\
Disminuyó & 8 & 44,4 \\
\hline TOTAL & 18 & 100,0 \\
\hline
\end{tabular}

*Dos participantes abandonaron
Tabla 5. Efectividad del mate de coca para disminuir el estrés laboral

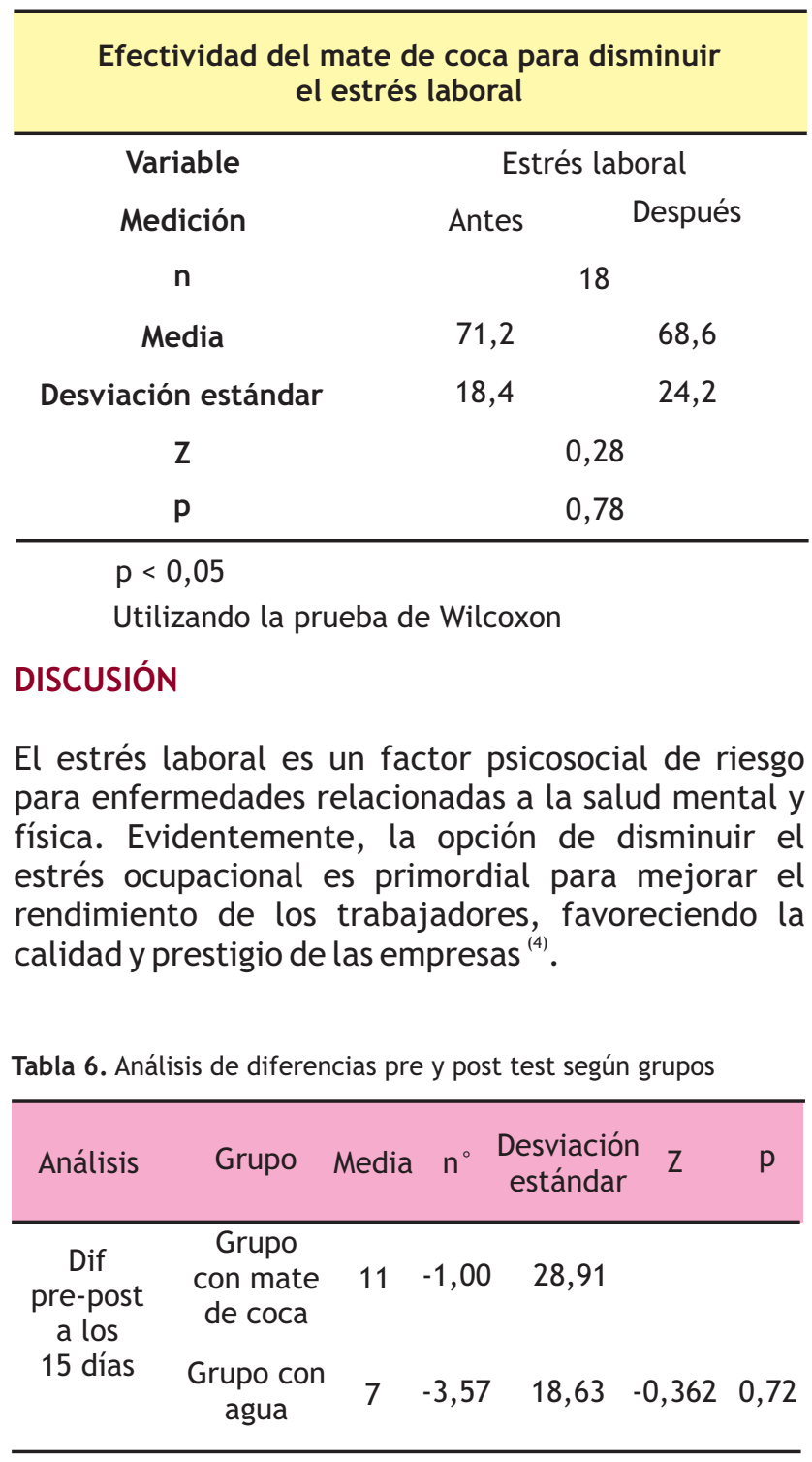

\section{U de Mann Whitney}

Desde el punto de vista de salud ocupacional, es una utopía erradicar el estrés relacionado al trabajo, por lo que diferentes centros de labores tienen el verdadero desafío de desarrollar medidas para minimizarlo y que aquél no desencadene una eventual enfermedad ocupacional.

Dentro de la medicina tradicional, existen elementos útiles para mejorar la calidad de las personas y trabajadores, como la hoja de coca. Es evidente, que el chacchado de la hoja puede no ser del agrado de muchos, sobre todo en los lugares donde se realiza 
trabajo administrativo. Incluso, es sabido que en nuestra sociedad, algunos tienden a rechazarlo. Pero una medida ampliamente aceptada, cómoda, asequible, económica y útil es el uso de infusiones como el mate de coca en los centros de labores.

Desde la antigüedad, uno de los beneficios encontrados de la hoja de coca es en la fatiga laboral, utilizándola empíricamente en diferentes trabajos que requieren esfuerzo físico ${ }^{(13)}$; sin embargo, se desconoce su uso en labores que generan esfuerzo mental, como el trabajo administrativo que desarrollan las personas en las distintas instituciones, como en las universidades. Teniendo en cuenta que es un trabajo demandante, ellos se enfrentan no solo a jefes y compañeros de trabajo, sino al cliente externo, caracterizado por grupos de estudiantes y padres de familia, los que indudablemente generan una respuesta de estrés, que si no es bien controlada por el sujeto, contribuiría a efectos graves, como el famoso síndrome de Burnout ${ }^{(24)}$.

Al conocer la importancia de controlar el estrés ocupacional, se buscaron diferentes intervenciones, encontrándose una opción en el uso de la infusión de las hojas de coca. Ellas tienen como compuesto primordial a la cocaína, que encontrada en el plasma equivale al $0,5-1,5 \%$ cuando se le ingiere como mate de coca y poseen un efecto corto, de hasta una hora, por lo que el margen de seguridad es amplio, sin ser asociado a dependencia ${ }^{(16)}$. De este modo, elegirlo como posible reductor de estrés fue una alternativa interesante e inédita.

Se aplicó el cuestionario validado para la detección de estrés laboral de la OIT/OMS utilizando la mediana 51 como punto de corte, además de unas preguntas estructuradas para el conocimiento de las características sociodemográficas de la población que ingresó al estudio.

En estudios que evaluaron la prevalencia de estrés laboral, se encontraron que podría alcanzar hasta el $40 \%$, lo que correspondería a lo encontrado en esta investigación ${ }^{(25)}$. Esto puede ser debido a que al seleccionar al personal administrativo como población de estudio y no al universo de trabajadores de la FMH - USMP, la proporción no representaría a toda la institución, siendo posiblemente menor. Por otro lado, por ser considerados los administrativos como grupo de riesgo por factores psicosociales, podría evidenciarse el similar porcentaje de estrés laboral con empresas conocidas con altos niveles ${ }^{(26)}$. De todos modos, el encontrar que casi $50 \%$ de los administrativos tienen estrés laboral es un dato importante que requeriría tomar medidas inmediatas. De los 20 individuos que ingresaron al estudio, correspondieron en su mayoría $(n=9)$ al grupo etario de 20 a 40 años. Esto indicaría que, existe mayor relación de estrés laboral con los grupos de menor edad, lo que se relaciona con la literatura, donde indican que el estrés aparece como la tercera causa de problemas de salud en trabajadores de 25 a 34 años ${ }^{(27,28)}$.

Con respecto al tiempo promedio de trabajo por día, el $90 \%$ que trabaja más de 8 horas tiene estrés laboral, asociando el mayor tiempo de trabajo por día a mayor estrés. Los turnos y horas de trabajo prolongados están descritos como condiciones que causan estrés, por lo que trabajar más allá de las horas de trabajo, no contribuye a una calidad de vida adecuada ${ }^{(29)}$. Por otro lado, llama la atención que a pesar que un porcentaje de los administrativos realiza técnicas de relajación (meditación y actividad física), presentan estrés laboral.

En los 18 administrativos que finalizaron el experimento, se encontró que el nivel de estrés laboral luego de tomar el mate de coca en el período establecido (15 días en cada grupo), aumentó en un $50 \%$, disminuyó en un $44,4 \%$ y permaneció igual en un $5,6 \%$. Esto probablemente, porque el mate de coca no actuó de igual forma en todos los individuos. La farmacodinamia de cada persona es diferente, por lo que los efectos en el organismo, también lo son ${ }^{(25)}$, aunque sería necesario identificar niveles del metabolito para determinar con mayor precisión.

Al comparar el uso del mate de coca, antes y después con la prueba Wilcoxon, se aprecia un cambio en los puntajes promedio de estrés laboral (Tabla 5). Antes de la intervención la media era 71,2 y luego de la intervención, la media del puntaje llegó a 68,6. Estadísticamente, la diferencia observada no es significativa $(p>0.05)$, debido probablemente, a que la cantidad de individuos fue pequeña; sin embargo, sí tiene significancia clínica, por lo que lo percibido por los usuarios no se debería dejar de lado.

Se comparó a los dos grupos (experimento y control) en el pre y post test, con la prueba U de Mann Whitney (Tabla 6). Se observó que existe mayor diferencia pre y post test en la disminución del estrés laboral en el grupo control, que en el grupo con mate de coca, sin significancia estadística $(p>0.05)$, por el probable efecto placebo. 
A pesar de las diferentes limitantes (el no uso de análisis laboratoriales, el corto tiempo del estudio, el poco número de participantes que influyó posiblemente en la significancia estadística, que a su vez, no permitiría extrapolar lo encontrado al resto de trabajadores, entre otras), resalta la importancia de haber iniciado las posibles intervenciones en salud ocupacional de la mano de la medicina tradicional, dado que nunca se había realizado este tipo de estudio.

En conclusión, el mate de coca no fue efectivo en la reducción del estrés laboral en los administrativos de la Facultad de Medicina Humana de la Universidad de San Martín de Porres - Lima, en el Semestre 2014 - II. Se recomienda desarrollar estrategias de manejo y afrontamiento del estrés laboral, teniendo como objetivos a la organización y el individuo, tomando como ejemplo las intervenciones para el control del burnout y agotamiento laboral, estimulando áreas que muchas veces no se toman en cuenta, como la despersonalización y logros personales. Además, del desarrollo de guías y capacitaciones regulares sobre salud mental en el trabajador, dando mayor importancia a la psicología ocupacional para la adopción de medidas necesarias ${ }^{(30)}$.

La investigación sobre intervenciones asequibles y útiles que se puedan aplicar en el ambiente de trabajo es necesaria y altamente recomendable, utilizando un mayor número de individuos de estudio, tiempo de seguimiento y apoyo diagnóstico de laboratorio e imágenes, tomando en consideración a la medicina tradicional como posible herramienta de intervención, para así contribuir con la salud de los trabajadores.

\section{REFERENCIAS BIBLIOGRÁFÍCAS}

1. Sandín B. El estrés. Análisis basado en el papel de los factores sociales. Rev. Internacional de Psicología Clínica y de la Salud. 2003, Vol. $3 \mathrm{~N}^{\circ} 1,141-157$.

2. Ivancevich J, Matteson M. Estrés y trabajo: Una perspectiva gerencial. $2^{a}$ edición. Editorial Trillas. México. 1989.

3. Selye $\mathrm{H}$. Confusion and controversy in the stress field. J Human Stress. 1975. Jun;1(2):37-44.

4. Leka S, Griffiths A, Cox T. La organización del trabajo y el estrés. Serie protección de la salud de los trabajadores $\mathrm{N}^{\circ} 3$. OIT - OMS. 2004.

5. Reglamento de la Ley $N^{\circ} 29783$, Ley de Seguridad y Salud en el Trabajo DECRETO SUPREMO Nº 005-2012-TR, y modificación con el Decreto Supremo N ${ }^{\circ} 006$ - 2014 - TR.

6. Hurtado de Mendoza C. Percepción de riesgos psicosociales, estrés, ansiedad, variables de salud y conciliación de la vida laboral-familiar en trabajadores y trabajadoras. Tesis Doctoral. España: Universidad Complutense de Madrid. 2013.

7. Rodríguez R, De Rivas S. Los procesos de estrés laboral y desgaste profesional (burnout): diferenciación, actualización y líneas de intervención. Med Segur Trab. 2011. Suplemento 1: $1-262$.

8. Gil P. Riesgos Psicosociales en el Trabajo y Salud Ocupacional. Rev Perú Med Exp Salud Pública. Perú. 2012; 29(2):237-41.

9. Perales A, Chue H, Padilla A, Barahona L. Estrés, ansiedad y depresión en magistrados de Lima, Perú. Rev Perú Med Exp Salud Pública. 2011.

10. Román J. Estrés y Burnout en profesionales de la salud de los niveles primario y secundario de atención. Rev Cubana Salud Pública. Ciudad de La Habana. 2003.

11. Guerrero E. 2003. Análisis pormenorizado de los grados de burnout y técnicas de afrontamiento del estrés docente en profesorado universitario. España: Universidad de Murcia. Anales de Psicología 2003. 19 (1), 145-158.

12. Takahashi M. Prioritizing sleep for healthy work schedules. Journal of Physiological Anthropology. Revisión 2012. Encontrado el 18 de setiembre del 2015. Disponible en: http://www.jphysiolanthropol.com/content/31/1/6

13. Llosa T, Chang E, Flores E, Dongo S, Luna L, Llosa L. Primer Estudio Psicofisiológico y Toxicológico de la Harina de Coca. Perú. T.Llosa (ed). Coca Médica. Año 1, № 1. 2006.

14. Cabieses F. Debate Agrario: La Hoja de Coca y sus Encrucijadas. Perú. 1996. Encontrado el 13 de junio 2014. Disponible en: http://www.cepes.org.pe/debate/debate25/08_Articulo.pdf

15. Rospigliosi F, Blondet C, Lloréns J. El consumo tradicional de la hoja de coca en el Perú. Perú: IEP Ediciones. 2004, 133 - 134.

16. Morera F, Ripoll F, Martín J, Checa F. Colinesterasa plasmática como indicador de gravedad en procesos agudos. Rev. Medicina Clínica. 2005;124(11);26.

17. Siegel R, Elsohly M, Plowman T, Rury P, Jones R. Cocaine in Herbal Teal. JAMA 255 (1): 40. 1986.

18. Rush C, Baker R, Wright K. Acute physiological and behavioral effects of oral cocaine in humans. Rev Drug Alcohol Depend. 1999. 1; 55 (1-2): 1-12.

19. Walsh S, Haberny K, Bigelow G. Modulation of intravenous cocaine effects by chronic oral cocaine in humans. Rev. Psychopharmacology (Berl). 2000; 150(4): 361-73.

20. Lizasoain I., Moro M., Lorenzo P. Cocaína: aspectos farmacológicos. España: Universidad Complutense de Madrid. Rev. Adicciones. 2002. 14(1):57-64.

21. Velásquez $Y$, Hernández $Y$, Rodríguez L, Morales A, Rincón J, Ramírez L. Detección de Benzoilecgonina en orina de consumidores de té de coca mediante métodos de inmunoensayo. INHRR. N 1 Caracas. 2011.

22. Suárez A. Adaptación de la Escala de estrés laboral de la OIT OMS en trabajadores de 25 a 35 años de edad de un Contact Center de Lima. Revista PsiqueMag. Junio 2013. Encontrado el 2 de abril del 2015. Disponible en: http://www.ucvlima. edu.pe/psiquemag/index.html

23. Carreño S, Medina M, Ortiz A, et. al. Factores psicosociales, consumo de alcohol y problemas laborales en trabajadores de una empresa química. La Psicología Social en México. 2002.

24. Aranda C, Pando M, Pérez M. Apoyo social y síndrome de quemarse en el trabajo o burnout: una revisión. Psicología y Salud. 2004;14(1):79-87.

25. Velázquez L, Lorenzo P, Moreno A. Farmacología Básica y Clínica. $18^{\circ}$ edición. Madrid, España. Editorial Médica Panamericana. 2008.

26. Chacín B, Corzo G, Rojas L, Rodríguez E, Corzo G. Estrés organizacional y exposición a ruido en trabajadores de la planta de envasado de una industria cervecera. Invest. Clín. 2002;43(4).

27. Instituto Nacional de Seguridad e Higiene en el Trabajo. V Encuesta Nacional de Condiciones de Trabajo. 2003. Revisión online. Encontrado el 13 de setiembre 2015. Disponible en: http: //www.oect.es/Observatorio/Contenidos/InformesPropi os/Desarrollados/Ficheros/Informe_V_ENCTDic2010.pdf 
Efectividad del uso del mate de coca en la reducción del estrés laboral en administrativos de una universidad

28. Osca A, Segado S, García, J. Inclusión social, mercado de trabajo y salud laboral: perspectivas sobre el estrés laboral de los jóvenes españoles. En el Portal del Instituto de la Juventud. 2006. Encontrado el 10 de octubre de 2015. Disponible en: http://www.injuve.es/sites/default/files/capitulo4_0.pdf

29. Instituto Nacional para la Seguridad y Salud Ocupacional. El estrés en el trabajo. CDC. 1999. Encontrado 4 de setiembre del 2015.Disponible en: http://www.cdc.gov/spanish/ niosh/docs/99-101_sp/

30. Maricutoui L, Sava F, Butta O. The effectiveness of controlled interventions on employees' burnout: A metanalisis. Journal of Occupational and Organizational Psychology. 2014.

\section{Fuentes de financiamiento}

Este artículo ha sido financiado por la autora.

\section{Conflictos de interés}

La autora declara no tener ningún conflicto de interés.

\section{Correspondencia:}

María Teresa Rivera Encinas

Dirección: Av. Alameda del Corregidor № 1531, La Molina. Teléfono: 3652300

Correo electrónico: mriverae@usmp.pe

Recibido: 29 de enero de 2016

Aprobado: 02 de agosto de 2016 